\title{
Heavy mesons in the Quark Model
}

\author{
D.R. Entem ${ }^{1, *}$, P.G. Ortega ${ }^{1}$, J. Segovia ${ }^{2}$, and F. Fernández ${ }^{1}$ \\ ${ }^{1}$ Grupo de Física Nuclear and Instituto Universitario de Física Fundamental y Matemáticas (IUFFyM), \\ Universidad de Salamanca, E-37008 Salamanca, Spain \\ ${ }^{2}$ Grup de Física Teòrica, Dept. Física and IFAE-BIST, Universitat Autònoma de Barcelona, E-08193 \\ Bellaterra (Barcelona), Spain
}

\begin{abstract}
Since the discovery of the $J / \psi$, the quark model was very successful in describing the spectrum and properties of heavy mesons including only $q \bar{q}$ components. However since 2003, with the discovery of the $X(3872)$, many states that can not be accommodated on the naive quark model have been discovered, and they made unavoidable to include higher Fock components on the heavy meson states. We will give an overview of the success of the quark model for heavy mesons and point some of the states that are likely to be more complicated structures such as meson-meson molecules.
\end{abstract}

\section{Introduction}

In November 1974 the Brookhaven National Laboratory [1] announced the discovery of a new particle, called $J$. Almost at the same time the existence of another new particle, called $\psi$, was reported by the Stanford Linear Accelerator [2]. Both particles shared similar properties and were interpreted as a $q \bar{q}$ state made of a new quark, the charm quark, previously predicted by the GIM mechanism [3] which explained the suppression of flavor changing kaon weak decays. These discoveries started a fast development of particle physics that is known as the November Revolution.

Very soon after, the $\Upsilon(1 S)$ was discovery at Fermilab [4] at higher energies confirming the existence of a fourth quark, the bottom quark. Until 1980, 11 states were collected by the Particle Data Group in these energy ranges [5] (see Table 1).

In 1978 the Cornell model [6] was developed and it already took into account the most important features of the quark-antiquark interaction in the heavy sector. The basic assumptions of the model were that the interactions were governed by $S U(3)$ color gauge symmetry with flavor only broken by the quark masses. It includes a coulomb term, induced by a onegluon exchange interaction, and a phenomenological confining interaction, that was taken to be linear. The interactions were flavor independent and spin independent, implementing the well known nowadays Heavy Flavor Symmetry and Heavy Quark Spin Symmetry.

The original naive quark model from Cornell was very successful in explaining the charmonium and bottomonium spectrum [7]. The model parameters were fitted to the 11 states mentioned before. The model gave predictions mainly for the bottomonium spectrum and comparing with the data of the PDG 2003 [8], where 15 states were added, all the predictions were on the correct energy range. In order to give a better description of the spectrum

\footnotetext{
*e-mail: entem@usal.es
} 
Table 1. States included in the Particle Data Group in 1980 [5].

\begin{tabular}{lll}
\hline & Mass $(\mathrm{MeV})$ & $J^{P}$ \\
\hline$J / \psi(3100)$ & $3097 \pm 1$ & $1^{-}$ \\
$\chi(3415)$ & $3414 \pm 4$ & $0^{+}$ \\
$p_{c}$ or $\chi(3510)$ & $3507 \pm 4$ & \\
$\chi(3550)$ & $3551 \pm 5$ & \\
$\psi(3685)$ & $3685 \pm 1$ & $1^{-}$ \\
$\psi(3770)$ & $3768 \pm 3$ & $1^{-}$ \\
$\psi(4030)$ & $4030 \pm 6$ & $1^{-}$ \\
$\psi(4160)$ & $4159 \pm 20$ & $1^{-}$ \\
$\psi(4415)$ & $4415 \pm 6$ & $1^{-}$ \\
$\Upsilon(9460)$ & $9458 \pm 6$ & $1^{-}$ \\
$\Upsilon(10020)$ & $10016 \pm 14$ & $1^{-}$ \\
\hline
\end{tabular}

more elaborate models were developed being some of the most representative the model of Godfrey and Isgur [9] and the model of Ebert et al. [10].

The situation changed completely in 2003 with the discovery of the $X(3872)$ by the Belle Collaboration [11] and very soon after confirmed by the CDF [12], D0 [13] and BaBar [14] Collaborations. This state could decay into $J / \psi \rho$, an isospin 1 channel, which ruled out completely a pure $c \bar{c}$ interpretation. Its closeness with the $D^{0} \bar{D}^{* 0}$ threshold suggested a molecular interpretation and clearly showed that the naive quark model was not enough to explain this state.

The number of states on the charmonium and bottomonium region has increase since 2003 and in the PDG 2017 [15] a total of 57 states are quoted, some of them with a clear non- $q \bar{q}$ nature.

\section{Two meson dynamics and the Chiral Quark Model}

In order to understand the $X(3872)$ the two meson dynamics is necessary. The first thing to notice is that quark models for heavy mesons only include color interactions. The interaction generated between mesons for such interactions between quarks cancels since mesons are colorless objects. However in the case of open charm meson-antimeson states the interaction between light quarks is also relevant.

In the light quark sector another important feature of QCD arises. The QCD Lagrangian is Chiral symmetric for massless quarks. However Chiral symmetry is not realized in the light meson sector, and the small masses of light quarks can not explain the breaking observed. This breaking is understood due to the spontaneously symmetry breaking of the QCD vacuum which generates the appearance of Goldstone bosons. For exact symmetry the Goldstone bosons are massless, however in the case of two flavor QCD they are light bosons which we can clearly identify with pions. The important point here is that now light quarks can interact through the exchange of Goldstone bosons, and these interactions are colorless, so they don't cancel between colorless objects. This is a way to build the two meson dynamics from the quark interaction.

With these ideas the Chiral Quark Model was developed [16] and extended to $S U(3)$ [17]. It has extensively used in the literature to study the $N N$ interaction [18], the baryon spectrum [19] and the meson spectrum [17, 20, 21].

With the interaction between quarks we use the Resonanting Group Method (RGM) [22] to obtain the interaction between mesons (diagrams on the first row of Fig. 1) or transitions 

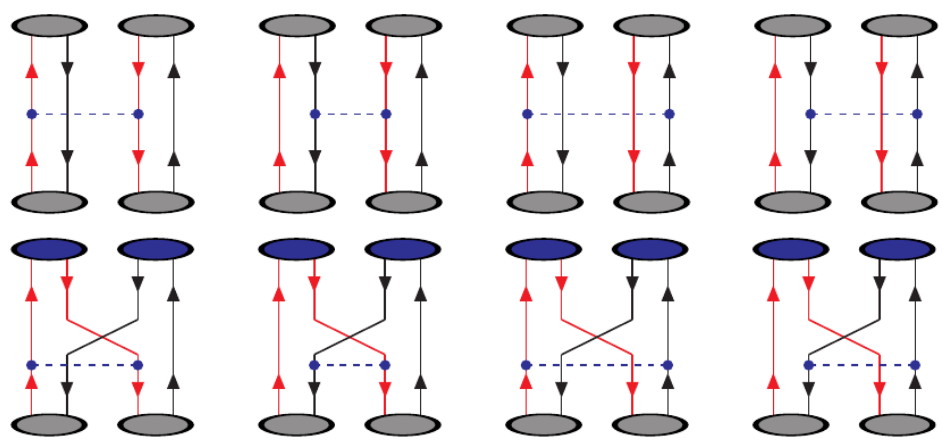

Figure 1. Diagrams that contribute to the interaction between mesons and rearrangement processes.

from a two meson channel to another (diagrams on the second row of Fig. 1), which are the so called rearrangement processes.

However the dynamics between two meson states is also governed by the coupling with one meson states. This is clear since one meson states decays strongly into two meson states through OZI allowed decays, and it was already taken into account in the original Cornell Model [6]. A simple an efficient way to account for such processes is to used a phenomenological ${ }^{3} P_{0}$ model [23]. It has been extensively used in different systems and it only depends on a parameter that can run with the scale [24].

With all this in mind one can start from a wave function

$$
|\Psi\rangle=\sum_{\alpha} c_{\alpha}|\psi\rangle+\sum_{\beta} \chi_{\beta}(P)\left|\phi_{M_{1}} \phi_{M_{2}} \beta\right\rangle,
$$

where the first term takes into account the relevant $c \bar{c}$ bare states and the second incorporates the two meson components.

One ends up with a Schrödinger type equation

$$
\sum_{\beta} \int\left(H_{\beta^{\prime} \beta}^{M_{1} M_{2}}\left(P^{\prime}, P\right)+V_{\beta^{\prime} \beta}^{e f f}\left(P^{\prime}, P\right)\right) \chi_{\beta}(P) P^{2} d P=E \chi_{\beta^{\prime}}\left(P^{\prime}\right)
$$

with

$$
V_{\beta^{\prime} \beta}^{e f f}\left(P^{\prime}, P\right)=\sum_{\alpha} \frac{h_{\beta^{\prime} \alpha}\left(P^{\prime}\right) h_{\alpha \beta}(P)}{E-M_{\alpha}}
$$

an effective potential between mesons due to the coupling with bare $q \bar{q}$ states, being $h_{\alpha \beta}(P)$ the vertex function given by the ${ }^{3} P_{0}$ model. Here it is important to notice that the states that give a higher contribution are those close to the threshold, being attractive when the state is above and repulsive when the state is below.

\section{Threshold effects in the heavy meson spectrum}

There are many states that can be accommodated in the naive quark model or given basically by a two meson molecule. However this is not always the case and an example is the already mentioned $X(3872)$. As explained before this state has properties that rules out a naive quark model interpretation, however it could be a two meson molecule. Its nature has important consequences since we have in hand important symmetries as Heavy Quark Spin 
Symmetry and Heavy Flavor Symmetry. This has been studied in the pure molecular picture in Refs. $[25,26]$. The unavoidable consequence is that the existence of the $X(3872)$ in the $1^{++}$sector implies the existence of a partner in the $2^{++}$channel in the charmonium sector, and the existence of their analogs in the bottomonium sector. None of this states have been found and in particular the bottom analog of the $X(3872)$ has been searched by the CMS [27], the ATLAS [28] and the Belle [29] Collaborations and no state was found.

This fact suggests that the state should have a different nature. In the energy region of the $X(3872)$ most quark models predict states with a higher mass around 3910. However the closeness of the $D D^{*}$ threshold implies that there should be a mixing between naive quark model states and two meson components. It has been shown in Ref. [30] that, in the Chiral Quark Model, a state near the $D^{0} D^{* 0}$ threshold appears when the coupling with the naive $\chi_{c 1}(2 P)$ state is considered, since this state is close enough to give a sizeable attraction. A similar calculation at baryon level has been carried out in Refs. [31, 32].

In this picture the consequences of HQSS and HFS are different. The model has these two symmetries [33] in the heavy sector. However the mass difference between the $D$ and $D^{*}$ mesons, which is around $100 \mathrm{MeV}$, plays an important role since the relative position between the naive quark model states and the relevant thresholds changes from one channel to the other. This energy region has been analyzed in detailed in Ref. [34]. There it was demonstrated that the $2^{++}$analog does not appear within the model. The naive quark model states get dressed but only an additional state is found in the $1^{++}$channel (the $\mathrm{X}(3872)$ ) an one in the $0^{++}$channel.

The $2^{++}$state is interesting because its decay properties suggest that the states $X(3915)$ and $X(3930)$ could be the same state with these quantum numbers. This situation agrees with Ref. [35].

In the $0^{++}$channel two states appear. In this channel there were originally two experimentally measured states, the $Y(3940)$ and the $X(3915)$, which later on were seen as the same by the community. It was even relabeled by the PDG as the $\chi_{c 0}(2 P)$ although this assignment has been abandoned. If the $X(3915)$ is the $2^{++}$state then one of the $0^{++}$could be the $Y(3940)$ and the other a recently measured resonance called $X(3860)$.

However, as mentioned before, threshold effects are not always essential. Recently the LHCb Collaboration measured four new resonances [36, 37], namely, the $X(4140), X(4274)$, $X(4500)$ and $X(4700)$, with quantum numbers $1^{++}$for the first two states and $0^{++}$for the last two states. As these states are well above threshold one would expect that the coupling with two meson states were very important. In Ref. [38] these states have been analyzed. Results indicate that the $X(4274)$ could be basically the $\chi_{c 1}(3 P)$ state with some dressing from nearby thresholds. The same situation is found for the $X(4500)$ that could be the $\chi_{c 0}(4 P)$ and the $X(4700)$ the $\chi_{c 0}(5 P)$. For the $X(4140)$, that could be a threshold cusp, no candidate has been found.

\section{Summary}

The naive quark model has been very successful at explaining the spectrum of heavy mesons in the charmonium and bottomonium regions. However with the discovery of the $X(3872)$ in 2003 it became clear that more complex structures than quark-antiquark states should be present. Although the naive quark model represents a good guidance to understand the spectrum, the mixing with nearby two meson thresholds have to be taken into account.

Some times the mixing only represents a dressing of naive quark model states, without a big change of their properties. However, in some cases, the mixing implies the existence of additional states with respect to the ones predicted by the naive quark model. 
On the other hand the picture for two meson molecules could be quite different when mixing with one meson states are taken into account. In particular expected symmetries as HQSS and HFS sometimes are not realized and results differ drastically from the expectations due to these symmetries.

This work has been partially funded by Ministerio de Economía, Industria y Competitividad under Contracts No. FPA2016-77177-C2-2-P, FPA2014-55613-P, FPA2017-86989-P and SEV-2016-0588 and by Junta de Castilla y León and ERDF under Contract No. SA041U16. P.G.O. acknowledges the financial support from Spanish MINECO's Juan de la Cierva-Incorporación programme, Grant Agreement No. IJCI-2016-28525. J.S. acknowledges the financial support from the European Union's Horizon 2020 research and innovation programme under the Marie Skłodowska-Curie Grant Agreement No. 665919, and from Spanish MINECO's Juan de la Cierva-Incorporación programme, Grant Agreement No. IJCI-2016-30028.

\section{References}

[1] J.J. Aubert et al., Phys. Rev. Lett. 33, 1404 (1974)

[2] J.-E. Augustin et al., Phys. Rev. Lett. 33, 1406 (1974)

[3] S.L. Glashow, J. Iliopoulos, and L. Maiani, Phys. Rev. D 2, 1285 (1970)

[4] S.W. Herb et al., Phys. Rev. Lett. 39, 252 (1977)

[5] R.L. Kelly et al., Rev. Mod. Phys. 52, S1 (1980)

[6] E. Eichten, K. Gottfried, T. Kinoshita, K.D. Lane, and T.-M. Yan, Phys. Rev. D 17, 3090 (1978)

[7] E. Eichten, K. Gottfried, T. Kinoshita, K.D. Lane, and T.-M. Yan, Phys. Rev. D 21, 203 (1980)

[8] K. Hagiwara et al., Phys. Rev. D 66, 010001 (2002)

[9] S. Godfrey, and N. Isgur, Phys. Rev. D 32, 189 (1985)

[10] D. Ebert, R. Faustov, and V. Galkin, Eur. Phys. J. C 71, 1825 (2011)

[11] S.-K. Choi et al. (Belle Collaboration), Phys. Rev. Lett. 91, 262001 (2003)

[12] D. Acosta et al. (CDF Collaboration), Phys. Rev. Lett. 93, 072001 (2004)

[13] V. Abrazov et al. (D0 Collaboration), Phys. Rev. Lett. 93, 162002 (2004)

[14] B. Aubert et al. (BaBar Collaboration), Phys. Rev. D 71, 071103 (2005)

[15] M. Tanabashi et al. (Particle Data Group), Phys. Rev. D 98, 030001 (2018)

[16] F. Fernández, A. Valcarce, U. Straub, and A. Faessler, J. Phys. G 19, 2013 (1993)

[17] J. Vijande, F. Fernández, and A. Valcarce, J. Phys. G 31, 481 (2005)

[18] D.R. Entem, F. Fernández, and A. Valcarce, Phys. Rev. C 62, 034002 (2000)

[19] A. Valcarce,P. González,F. Fernández, and V. Vento, Phys. Lett. B 367, 35 (1996)

[20] J. Segovia, A.M. Yasser, D.R. Entem, and F. Fernández, Phys. Rev. D 78, 114033 (2008)

[21] J. Segovia, P.G. Ortega, D.R. Entem, and F. Fernández, Phys. Rev. D 93, 074027 (2016)

[22] J.A. Wheeler, Phys. Rev. 52, 1083 (1937)

[23] L. Micu, Nucl. Phys. B 10, 521 (1969)

[24] J. Segovia, D.R. Entem, and F. Fernández, Phys. Lett. B 715, 322 (2012)

[25] J. Nieves, and M. Pavón-Valderrama, Phys. Rev. D 86, 056004 (2012)

[26] F.-K. Guo, C. Hidalgo-Duque, J. Nieves, and M. Pavón-Valderrama, Phys. Rev. D 88, 054007 (2013)

[27] CMS Collaboration, Phys. Lett. B 727, 57 (2013)

[28] ATLAS Collaboration, Phys. Lett. B 740, 199 (2015)

[29] X.H. He et al. (Belle Collaboration), Phys. Rev. Lett. 113, 142001 (2014) 
[30] P.G. Ortega, J. Segovia, D.R. Entem, and F. Fernández, Phys. Rev. D 81, 054023 (2010)

[31] E. Cincioglu, J. Nieves, A. Ozpineci, A.U. Yilmazer, Eur. Phys. J. C 76, 576 (2016)

[32] M. Takizawa, and S. Takeuchi, PTEP 2013, 093 D01 (2013)

[33] D.R. Entem, P.G. Ortega, and F. Fernández, AIP Conf. Proc. 1735, 060006 (2016)

[34] P.G. Ortega, J. Segovia, D.R. Entem, and F. Fernández, Phys. Lett. B 778, 1 (2018)

[35] T. Branz, R. Molina, and E. Oset, Phys. Rev. D 83, 114015 (2011)

[36] R. Aaij et al. (LHCb Collaboration), Phys. Rev. Lett. 118, 022003 (2017)

[37] R. Aaij et al. (LHCb Collaboration), Phys. Rev. D 95, 012002 (2017)

[38] P.G. Ortega, J. Segovia, D.R. Entem, and F. Fernández, Phys. Rev. D 94, 114018 (2016) 